\title{
Novel gratings for astronomical observations II
}

Noboru Ebizuka, Takayuki Okamoto, Yutaka Yamagata, Minoru Sasaki, Ichi Tanaka, et al.

Noboru Ebizuka, Takayuki Okamoto, Yutaka Yamagata, Minoru Sasaki, Ichi Tanaka, Takashi Hattori, Yusuke Nakauchi, Makio Nishimaki, Kazuya Yamamoto, Makoto Okada, Kazuto Saiki, "Novel gratings for astronomical observations II," Proc. SPIE 11852, International Conference on Space Optics — ICSO 2020, 1185263 (11 June 2021); doi: 10.1117/12.2600051

SPIE Event: International Conference on Space Optics - ICSO 2021, 2021, Online Only 


\section{International Conference on Space Optics-ICSO 2020}

Virtual Conference

30 March-2 April 2021

Edited by Bruno Cugny, Zoran Sodnik, and Nikos Karafolas
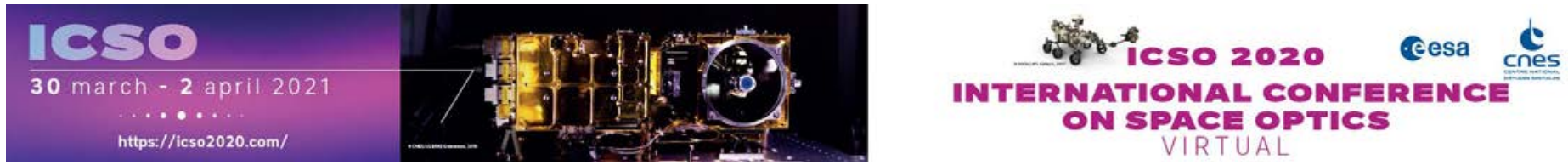

Novel gratings for astronomical observations II

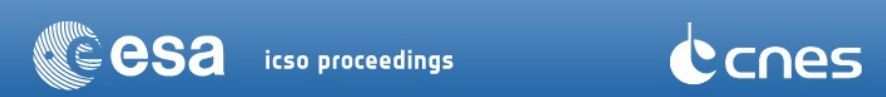

International Conference on Space Optics - ICSO 2020, edited by Bruno Cugny, Zoran Sodnik,

Nikos Karafolas, Proc. of SPIE Vol. 11852, 1185263 - @ 2021 ESA and CNES

CCC code: $0277-786 \mathrm{X} / 21 / \$ 21 \cdot$ doi: $10.1117 / 12.2600051$ 


\title{
Novel gratings for astronomical observations II
}

\author{
Noboru Ebizuka*a, Takayuki. Okamoto ${ }^{\mathrm{b}}$, Yutaka Yamagata ${ }^{\mathrm{a}}$, \\ Minoru Sasakic ${ }^{c}$ Ichi Tanaka ${ }^{d}$, Takashi Hattori ${ }^{d}$, Yusuke Nakauchie, \\ Makio Nishimaki ${ }^{\mathrm{f}}$, Kazuya Yamamoto ${ }^{\mathrm{f}}$ Makoto Okada ${ }^{\mathrm{f}}$ and Kazuto Saiki ${ }^{\mathrm{g}}$ \\ ${ }^{a}$ Center for Advanced Photonics, RIKEN, 2-1 Hirosawa, Wako, Saitama 351-0198, Japan, \\ ${ }^{\mathrm{b}}$ Advanced Device Laboratory, RIKEN, \\ ${ }^{\mathrm{c}}$ Faculty of Engineering, Toyota Technological Institute, \\ d Subaru Telescope, National Astronomical Observatory of Japan. \\ ${ }^{\mathrm{e}}$ Institute of Space and Astronautical Science, Japan Aerospace Exploration Agency, \\ ${ }^{\mathrm{f}}$ Engineering Development Department, Nalux Co. Itd., \\ ${ }^{\mathrm{g}}$ Graduate School of Science, Osaka University.
}

\begin{abstract}
Currently, we are developing a VB grating for an echelle grism of the Subaru Multi-Object InfraRed Camera and Spectrograph (MOIRCS) and a VB grating for the Advanced Lunar Imaging Spectrometer (ALIS) of the Lunar Polar Exploration Mission (LUPEX). The shapes of gratings are optimized to achieve high diffraction efficiency and a wide spectral bandwidth by performing numerical calculations of the rigorous coupled wave analysis (RCWA). Based on the calculated results, we are developing variety of gratings using MEMS technologies. In addition, we have developed new high-dispersion grisms of $\mathrm{J}$ and $\mathrm{H}$ band of MOIRCS with transmission gratings fabricated by LightSmyth. The transmission grating is kind of a VB grating which ridges are composed by three kinds of dielectric layers.
\end{abstract}

Keywords: Volume grating, Echelle spectroscopy, RCWA, MEMS technology

\section{INTRODUCTION}

Transmission gratings enable downsizing of the spectroscopic optical system and the entire instrument because the transmission grating can be placed near a collimator and imaging optical element. With such advantage, practical implementation of a transmission grating with high angular dispersion is expected for spectroscopic instruments of huge telescopes such as the $8.2 \mathrm{~m}$ Subaru telescope, TMT (Thirty Meter Telescope) and spaceborne telescopes, as well as planets and lunar explore [1-4].

Surface relief (SR) gratings of the transmission type with ridges of the conventional sawtooth shape are widely used for low dispersion spectrographs. However the diffraction efficiency of a SR grating decreases steeply as angular dispersion increases. A volume phase holographic (VPH) grating is a thick diffraction grating which refractive index is modulated sinusoidally. The VPH grating is able to achieve diffraction efficiency of up to $100 \%$ for $s$ - or $p$-polarization $[5,6]$, and are installed in many astronomical instruments. The authors had developed many VPH grisms for observational instruments of the $8.2 \mathrm{~m}$ Subaru Telescope, $1.88 \mathrm{~m}$ Telescope of the Okayama Astrophysical Observatory and other telescopes [7, 8]. However, a VPH grating may suffer from a narrow spectral bandwidth when angular dispersion increases. Furthermore, since a VPH grating has low diffraction efficiency at higher diffraction orders, it cannot be using the echelle spectroscopic method.
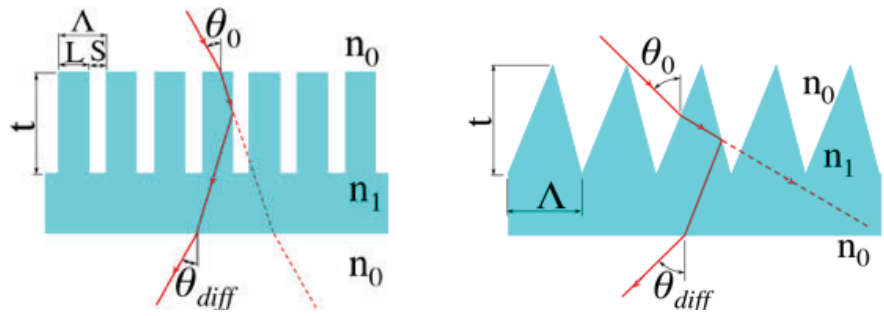

Figure 1. Schematic representation of VB grating (left) and RFT grating (right). 

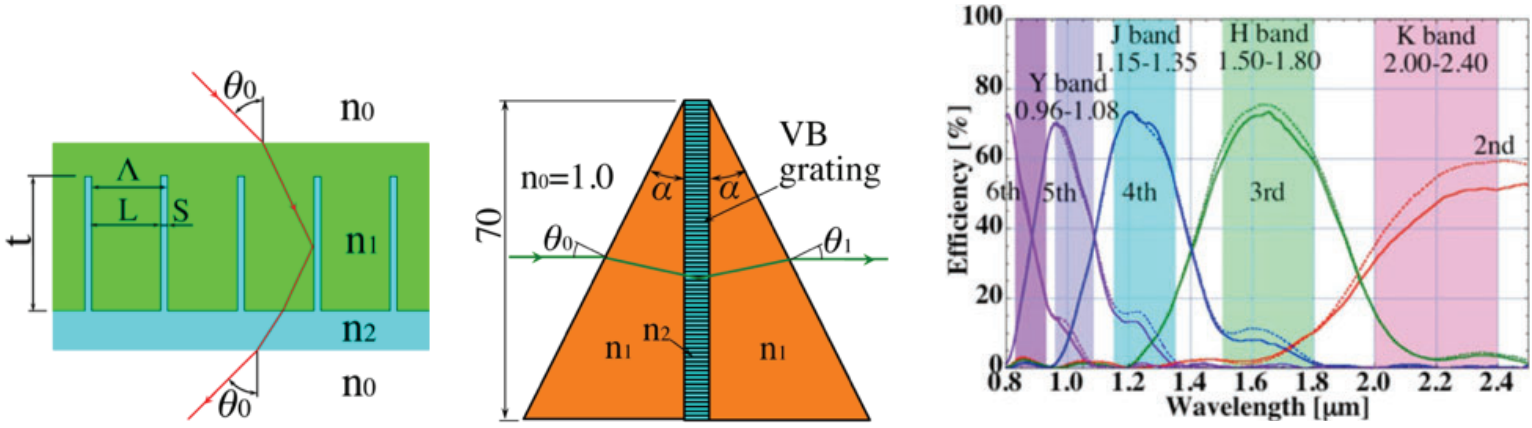

Figure 2. Schematic representation of VB grating (left), diffraction efficiency of VB grating for echelle grism of nu-MOIRCS (middle: $\theta_{0}=28.4^{\circ}, \mathrm{n}_{1}=1.33, \mathrm{n}_{2}=1.6, \Lambda=5.1 \mu \mathrm{m}, \mathrm{L} \& \mathrm{~S}=4.6: 0.5[\mu \mathrm{m}], \mathrm{t}=16 \mu \mathrm{m}$ ), and echelle grism (right) [18].

Both a VB (volume binary) grating (Fig. 1 left) [9, 10]and novel RFT (reflector facet transmission) grating (Fig. 1 right) $[11,12]$ are transmission gratings with large angular dispersion and high diffraction efficiency. We will introduce about RFT gratings next time, we describe the developments of VB gratings in this article.

\section{VB GRATING FOR ECHELLE GRISM OF MOIRCS}

The MOIRCS [13] is scheduled to upgrade to nuMOIRCS as the first light instrument for the ULTIMATE Subaru. The ULTIMATE Subaru plans to install a new ground-layer adaptive optics (GLAO) onto the Subaru telescope [14]. The GLAO improves the limiting magnitude and the spatial resolution by reducing the size of the star image by correcting the wavefront error due to the atmospheric turbulences. The echelle grism from the third to the sixth diffraction orders installs in the nuMOIRCS. The echelle grism combined with a direct vision prism as a cross disperser can simultaneously covert the wavelength range of 0.8 to $1.8 \mu \mathrm{m}$ with large angular dispersion by folding the spectra onto the detector, and it is one of the key optical-element to improve performance of the nuMOIRCS.

The VB grating uses the total reflection by a low-index medium as the mirror surface a (Fig. 2 left) $[11,15]$. The echelle grism is fabricated by sandwiched the VB grating between two ZnSe prisms as shown on the middle panel in Fig. 2. We found that higher diffraction efficiencies can be achieved by the VB grating (lattice period $\Lambda=5.1 \mu \mathrm{m}, \mathrm{L} \& \mathrm{~S}=4.6: 0.5[\mu \mathrm{m}$ ], thickness $\mathrm{t}=16 \mu \mathrm{m}$ ) for the echelle grism by numerical calculations using the RCWA $[16,17]$ method as shown on the right panel in Fig. 2. We decided to develop a VB grating for the echelle grism of the nuMOIRCS.

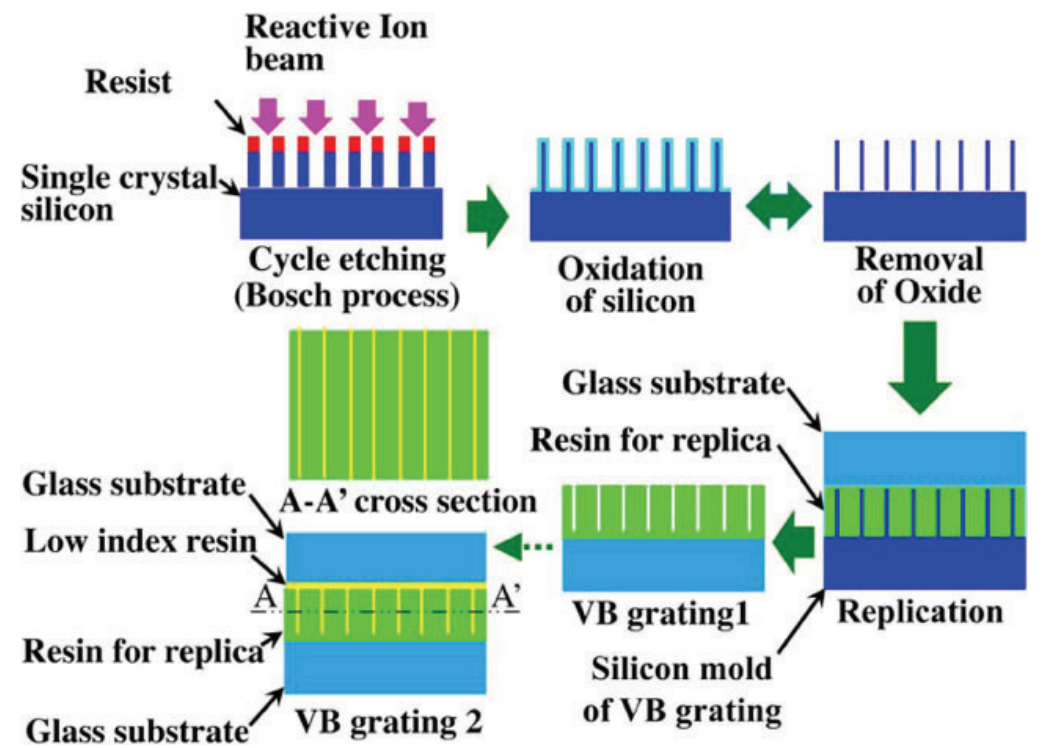

Figure 3. Fabrication process for replica VB grating by using silicon mold [18]. 

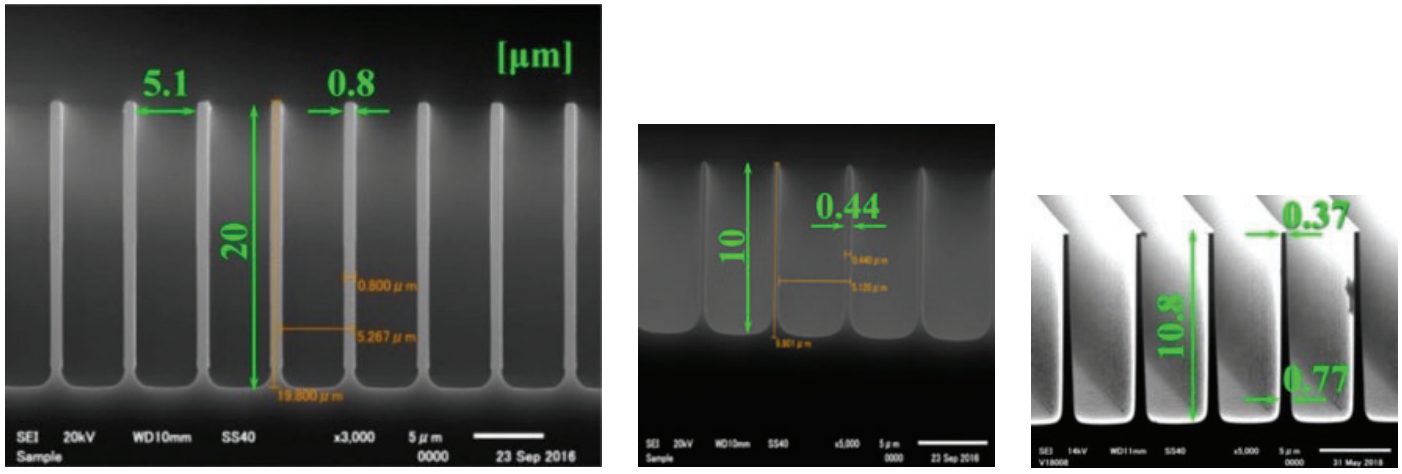

Figure 4. Silicon high aspect gratings as a mold.

\subsection{Silicon mold for replica of VB grating}

We develop a fabrication method for a VB grating as a mold of silicon with ridges of high aspect ratio in Toyoda Institute of Technology. By numerous trial fabrications, we found the process as shown in Fig. 3 which oxygen is slightly added to the etching gas and the passivation gas, cleaning is performed while etching and sidewall protection (Bosch process), and the amount of an etching volume is reduced. As a result, the scallops (irregularities formed every etching cycle) on the sidewall of the ridges successfully reduced to about $40 \mathrm{~nm}$. Further, silicon oxide layers are formed on both sides of the ridge surfaces of the silicon, and then the oxide layers are etched. By repeating this process several times, it was possible to obtain extremely smooth surfaces with little scattering even in visible light.

Specifically, a resist pattern was formed on a silicon substrate by using a mask with a line \& space of $2.0 \mu \mathrm{m}: 3.1 \mu \mathrm{m}$. Deep grooves formed by the cycle etching onto the silicon substrates (two kind of depths with $10 \mu \mathrm{m}$ and $20 \mu \mathrm{m}$ ). Silicon oxide layers are formed and removed twice. Where oxygen diffuses from a surface of the silicon is about $400 \mathrm{~nm}$ by the oxidation process when an oxide layer having a thickness of about $1 \mu \mathrm{m}$. As a result, lattices of ridges with widths of 0.44 and $0.8 \mu \mathrm{m}$, respectively, which surfaces are extremely smooth, are obtained (Fig. 4 left and middle).

The silicon VB grating with tapered grooves on the right panel in Fig. 4 is suitable for mold. Although the etching and sidewall protection process of the tapered VB grating are almost the same as the other VB gratings in Fig. 4 applied voltage or gas pressure is slightly different. We are performing replication tests by using the tapered VB grating.

\subsection{Silica VB grating [18]}

As for the new fabrication method of a VB grating, a prototype was fabricated by oxidizing the entire silicon of a highaspect-ratio rectangular grating, which is formed on a silicon on quartz (SOQ) wafer. The SOQ wafer is composed a thin silicon wafer bonded to a quartz glass wafer. We devised a fabrication method using a SOQ (silicon on quartz) substrate as shown in Fig. 5 [18]. The SOQ substrate consists of a thin silicon substrate bonded onto a quartz glass substrate. The silicon substrate was bonded to quartz glass substrate at Nanotech PF, and we commissioned a company to polish the

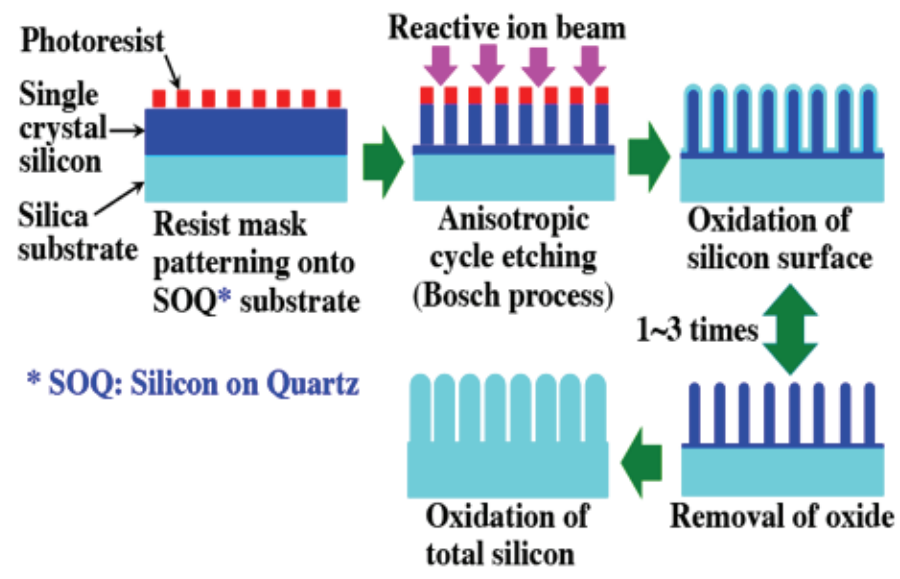

Figure 5. Fabrication method of silica VB grating using SOQ substrate [18]. 


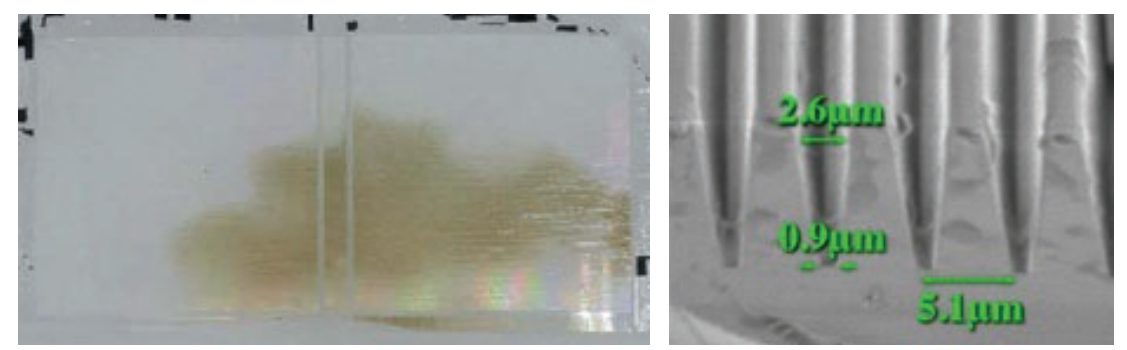

Figure 6. Photograph of prototype silica VB grating (left) and SEM photograph of grooves tilted with $45^{\circ}$ (right) [18].

silicon side. A silicon rectangular grating in the silicon substrate is manufactured in the same manner as the silicon mold described above, is further oxidized to form a silica VB grating. After number of trial and error, a silicon rectangular grating could be fabricated in a SOQ substrate. A silica VB grating was obtained by further oxidization of the silicon rectangular grating. As a result of observing diffracted light at an angle of incidence of $45^{\circ}$ by irradiating with a laser of $633 \mathrm{~nm}$, although ghost (horizontal direction) and flare (vertical direction) are seen, the laser intensity concentrates in the position at diffraction orders. We confirmed that the grating is functioned as a VB grating.

\section{VB GRATING OF GLASS FOR ALIS}

\subsection{VB grating of quartz glass}

We developed a VB grating of quartz glass for the Advanced Lunar Imaging Spectrometer (ALIS) of the Lunar Polar Exploration Mission (LUPEX) [4] which is $\mathrm{L} \& \mathrm{~S}=0.73 \mu \mathrm{m}: 0.73 \mu \mathrm{m}$ and $2.65 \mu \mathrm{m}$ in depth of grooves fabricated by anisotropic etching at Nalux Co. Ltd. Although grooves are slightly bowing shape, measured diffraction efficiency is almost identical with the designed value.

\subsection{VB grating of Tempax glass}

We are developing a fabrication method for a VB grating of Tempax (Borosilicate glass of Schott Corp.) at the TTI and Nalux Co. Ltd. because the aspect ratio of grooves of the VB grating will be increased by changing the InGaAs imaging detector for ALIS. The Tempax is excellent resistance to radio activity and high temperature.

The fabrication method for a Tempax VB grating will be described in detail with reference to Fig. 8. A line and space of resist is patterned on a silicon substrate by a laser exposure device or laser interference exposure, and a lattice of deep grooves is processed by cycle etching (Bosch process). By repeating the thermal oxidation of silicon and the removal of the oxide film by wet etching, the width of the ridges becomes narrower, and width of ridges or grooves is precisely adjusted. Further, the unevenness (scallop) generated in the cycle etching step is removed by the steps of oxidation and oxide film removal, and an optically smooth surface can be obtained.

The silicon mold is bonded with Tempax wafer by anode bonding in vacuum. Tempax is possible to attach by anode bonding with silicon because the glass wafer contains $\mathrm{Na}$ or other metal component and the glass has the same thermal expansion coefficient as silicon used for a mold, although when plasma etching processing is performed on borosilicate glass such as Tempax, it is difficult to process deep grooves because the contents such as $\mathrm{Na}$ and $\mathrm{Ca}$ are precipitated and etching is hindered.
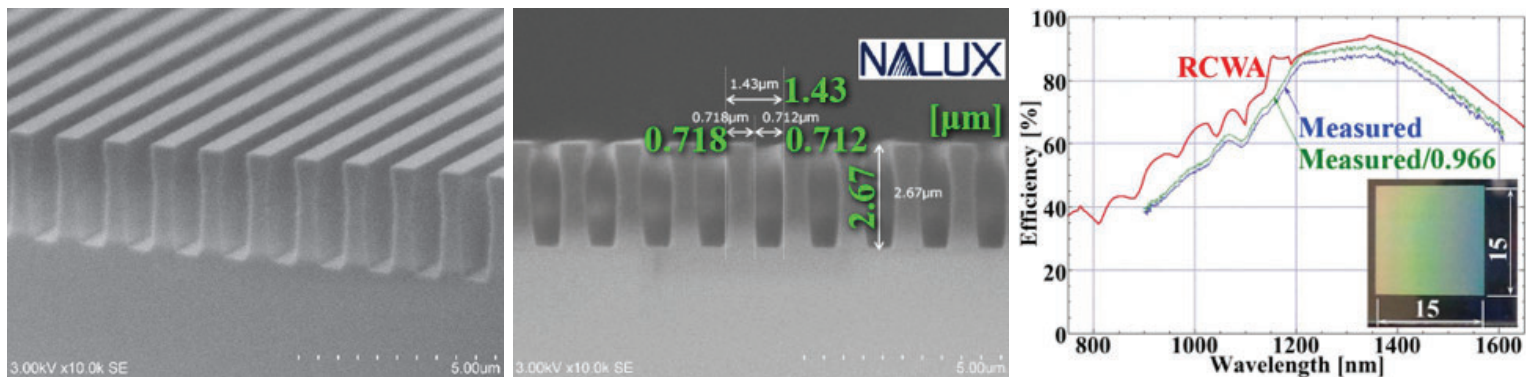

Figure 7. SEM photograph (left and middle) and diffraction efficiency (right) of VB grating designed for ALIS, $\theta_{0}=25.7^{\circ}$, $\mathrm{n}_{0}=1.0, \mathrm{n}_{1}=1.435, \Lambda=1.43 \mu \mathrm{m}$ with $\mathrm{L}: \mathrm{S}=1: 1, \mathrm{t}=2.65 \mu \mathrm{m}$. 


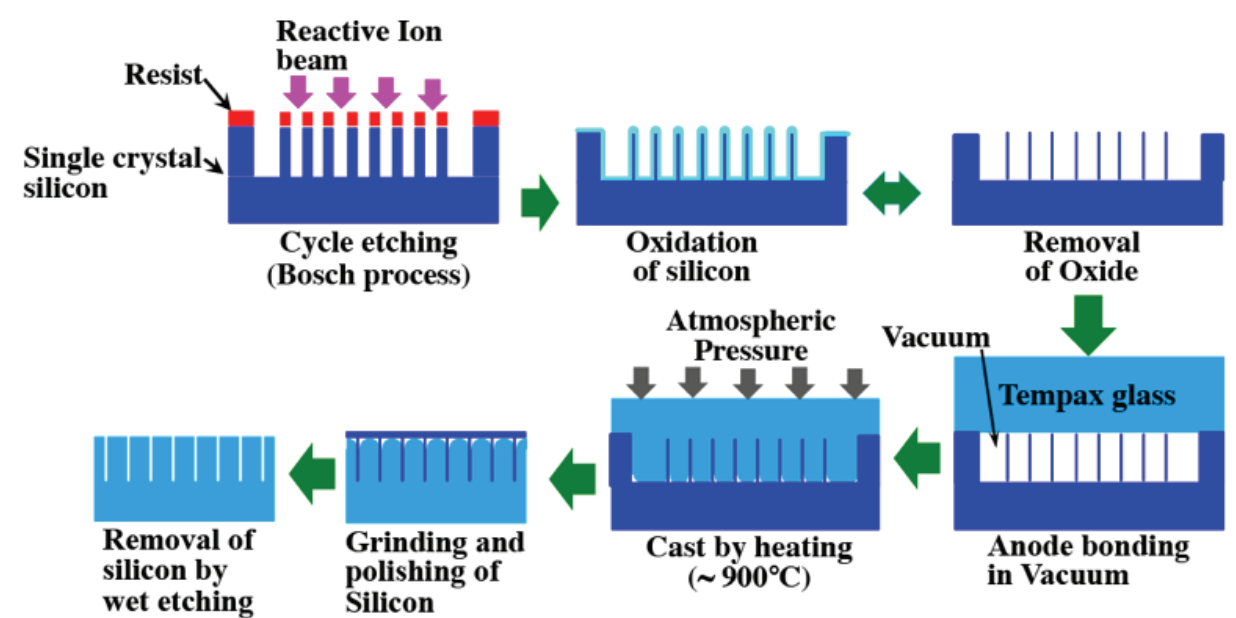

Figure 8. Fabrication method for VB grating of Tempax glass by using silicon mold.

When heated above the softening point of the glass material $\left(820^{\circ} \mathrm{C}\right.$ for Tempax $)$, the Tempax is casted into a silicon mold by atmospheric pressure. The silicon substrate and surface of Tempax wafer are grinded and polished. The silicon buried in the groove of the glass is removed by wet etching to complete a monolithic Tempax VB grating. If necessary, a resin which a refractive index is different from that of glass is filled into grooves of the VB grating.

\section{HIGH DISPERSION GRISM FOR MOIRCS}

Since the high-dispersion grisms with a VPH grating developed for MOIRCS were designed for the long-slit observation, and the grism has a narrow bandwidth when a slit is opened at a position away from the center of the field of view (offset slit). In the case of the multi-slit observation, it may take some effort to analyze for spectrum of an offset slit because the diffraction efficiency of the VPH grating at both ends of the spectral band fluctuates greatly.

The transmission grating developed by LightSmyth is able to achieve a diffraction efficiency above $95 \%$ at the peak, and its spectral bandwidth is from 3 to 5 times wider than that of a VPH grating. The LightSmyth grating is a kind of a VB grating with ridges structured by three layers dielectric (Fig. 9 left) [19]. We had developed two types of high-dispersion grisms for nuMOIRCS which a LightSmyth grating is sandwiched by two ZnSe prisms (Fig. 9 right), as a successor to the VPH grism [20].

For the $\mathrm{J}$ band $(1,150 \sim 1,350 \mathrm{~nm})$ grism, the ready-made $900 \mathrm{~nm}$ band grating was used, so it was not optimal, but the diffraction efficiency of the grism is $85 \%$ at the peak and above $60 \%$ in the entire spectral band, on the other hand, the efficiency of the VPH grism was $80 \%$ at the peak and about $30 \%$ at both ends of the spectral band (Figure 10 left). The diffraction efficiency of the $\mathrm{H}$ band $(1,500 \sim 1,800 \mathrm{~nm})$ grism is $95 \%$ at the peak and above $85 \%$ in the entire spectral band, on the other hand, the efficiency of the VPH grism is $70 \%$ at the peak and about $10 \%$ at both ends of the spectral band (Fig. 10 right).
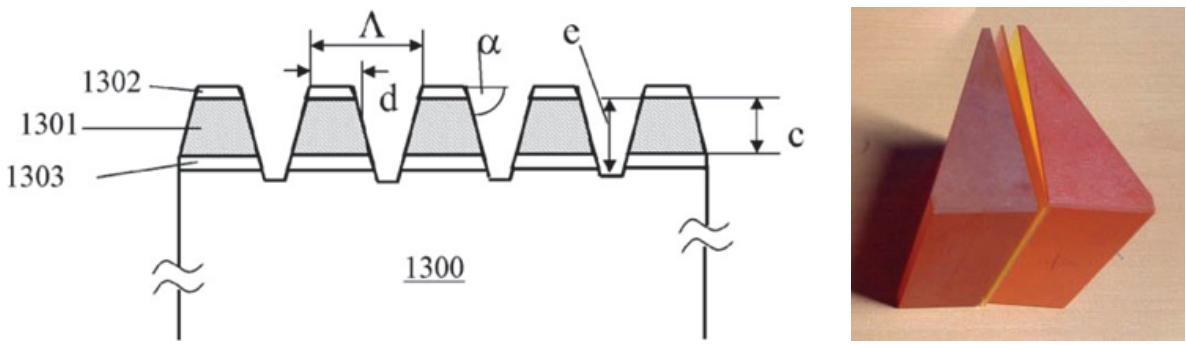

Figure 9. Schematic representation of transmission grating of LightSmyth (left) [19] and MOIRCS highdispersion grism with LightSmyth grating sandwiched by two ZnSe prisms (right). In the left panel, \#1300 is quartz glass, and \#1301, \#1302 and \#1303 are dielectrics. 

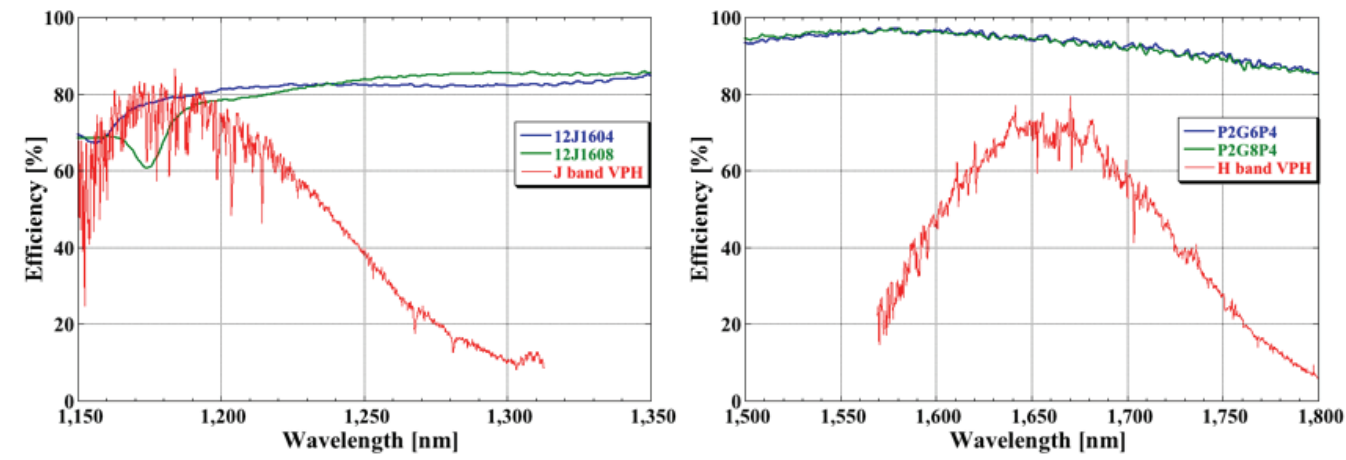

Figure 10. Measured diffraction efficiencies of J (left) and H (right) band grisms for MOIRCS. Blue and green lines indicate the new high-dispersion grisms with transmission grating of LightSmyth Co. Ltd., red lines indicate VPH grisms measured by on sky observations. The observation with the J band VPH grism had used offset slit.

\section{CONCLUSIONS}

VPH gratings have low diffraction efficiency at high diffraction orders and cannot be used with echelle Spectrographs. VB gratings, on the other hand, are used for echelle spectrographs. We are developing a VB grating for the echelle grism of MOIRCS which is fabricated by replication of a silicon mold, or by oxidization of silicon grating using SOQ substrate. In addition, we had developed high-dispersion grisms of MOIRCS with transmission gratings developed by LightSmith Co. Ltd. instead of VPH grisms. We are also developing the VB grating of the first diffraction order for ALIS which is fabricated by casting of Tempax glass into a silicon mold.

VB gratings are versatile dispersive-optical element for spectrometers that offer high resolving power and wide spectral bandwidth. A large amount of VB gratings are used for optical communications. Since a VB grating in monolithic quartz glass (silica) or Tempax glass is excellent resistance to radioactivity and high temperatures, it is well suitable for space applications as well as a pulse compressor for a high-power laser.

We appreciate Mr. Keizo Kajiura of Nanotechnology Platform facilities, the Toyota Institute of Technology for their assistance on the trial fabrications of the VB gratings. Professor Kodama of Tohoku University and Professor Motohara of the National Astronomical Observatory of Japan (NAO) / the University of Tokyo supported us for development of the new high-dispersion grisms for MOIRCS. We utilize facility of the Advanced Technology Center of NAOJ for grating measurements and "Nanotechnology Platform Japan" of the Ministry of Education, Culture, Sports, Science and Technology (MEXT), Japan for grating fabrications. This work was supported by the grant-in-aid of NAOJ for TMT strategic basic research and development, the Grant-in-Aid for Challenging Exploratory Research (15K13470) from MEXT, Kiban-A Grant-in-Aid for Scientific Research (18H03717) from MEXT and Adaptable and Seamless Technology Transfer Program through Target-driven R\&D from Japan Science and Technology Agency.

\section{REFERENCES}

[1] Simard, N.L., et al., "The instrumentation program for the Thirty Meter Telescope," Proc. SPIE 8446, 1F, (2012).

[2] Kevin. B., et al., "WFOS instrument trade study: slicer vs. fiber instrument concept designs and results," Proc. SPIE 10702, 20 (2018).

[3] Minowa, Y., et al., "An overview of current and future instrumentation at the Subaru telescope," Proc. SPIE 9908, 06 (2016).

[4] K. Saiki, et al., "Development of Two Types of NIR Spectral Camera for Lunar Missions SLIM and LUPEX," Proc. Lunar \& Planetary Sci. Conf. 52 (2021) in press.

[5] Barden, S.C., Arns; J.A. and Colburn, W.S., "Volume-phase holographic gratings and their potential for astronomical applications," Proc. SPIE 3355, 866-876 (1998).

[6] Baldry, I. K., Bland-Hawthorn, J. and Robertson, J. G., "Volume Phase Holographic gratings: Polarization properties and Diffraction Efficiency," PASP 116, 403-414 (2004).

[7] Ebizuka, N., et al., "Cryogenic VPH Grisms for MOIRCS," PASJ 63, S605-S612 (2011).

[8] Ebizuka, N., et al., "Grisms Developed for FOCAS," PASJ 63, S613-S622 (2011). 
[9] Gupta, M. C. and Peng, S. T., "Diffraction characteristics of surface-relief gratings," Appl. Opt. 32, 2911-2917 (1993).

[10] Gerritsen, H. J. and Jepsen, M. L., "Rectangular surface-relief transmission gratings with a very large first- order diffraction efficiency (95\%) for unpolarized light," Appl. Opt. 37, 5823-5829 (1998).

[11] Ebizuka, N., et al., "Novel gratings for next generation instruments of astronomical observations," Proc. SPIE 10233, 0M (2017).

[12] Ebizuka, N., et al., "Novel diffraction gratings for next generation Spectrographs with high spectral dispersion," Proc. SPIE 9912, 2Z01-2Z10 (2016).

[13] Ichikawa, T., et al., "MOIRCS: multi-object infrared camera and spectrograph for SUBARU," Proc. SPIE 6269, 16 (2006).

[14] Hayano, Y., et al., "ULTIMATE-SUBARU: project status," Proc. SPIE 9148, 2S01-2S08 (2014).

[15] Bianco, A. and Ebizuka, N., "Echelle VPHG: a step forward," Proc. SPIE 8450, 431-438 (2012).

[16] Moharam, M. G. and Gaylord, T. K., "Rigorous coupled-wave analysis of planar-grating diffraction," JOSA 71, $811(1981)$

[17] Li, L., "Note on the S-matrix propagation algorithm," JOSA A 20, 655 (2003).

[18] Ebizuka, N., et al. "Novel gratings for astronomical observation," CEAS Space Journal 12, 3-14 (2020).

[19] Mossberg, T. W., et al., "Highly efficient optical grating with reduced thickness requirements and impedancematching layers," US Patent: US 8,165,436 B2, (2008).

[20] Tanaka, I., et al., "Developing the wide-spectral coverage, very high-efficiency grisms for MOIRCS on Subaru Telescope" Proc. SPIE 11451, 5A1-11, (2020). 